\title{
Attitude, Practice and Associated Factors Towards Patients With Hepatitis B and C Viruses Among Nurses Working in Governmental Hospitals in Addis Ababa, Ethiopia, 2019.
}

\section{Tekalign Birlie Amera ( $\nabla$ tekalign1991@gmail.com )}

Lecturer of adult health nursing, Department of comprehensive nursing, college of health sciences, Debre Tabor university, Debre-Tabor, Ethiopia https://orcid.org/0000-0002-2378-0156

\section{Zeleke Argaw}

Addis Ababa University

\section{Teshome Habtie}

Addis Ababa University

Abraham Tsedalu

Debre Tabor University

\section{Research article}

Keywords: attitude, practice, hepatitis viruses B and C, nurses

Posted Date: October 14th, 2020

DOI: https://doi.org/10.21203/rs.3.rs-89376/v1

License: (9) This work is licensed under a Creative Commons Attribution 4.0 International License.

Read Full License 


\section{Abstract}

Background: nurses spend much time caring for patients with different diagnosis some of which are potentially infectious. Infectiousness of some diseases may affect nurse's behavior to care for patients equally and effectively. Hepatitis B and C are infectious global treats responsible for $96 \%$ of hepatitis related mortality. Hence, this study aimed to assess attitude, practice and associated factors towards patients with hepatitis $B$ and $C$ viruses among nurses working in governmental hospitals in Addis Ababa.

Methods: Institutional based cross-sectional study was conducted in public hospitals in Addis Ababa Ethiopia from April 01 to 30, 2019. A total of 396 nurses were selected using simple random sampling technique. Data was collected using a pretested, validated self-administered questionnaire. Epi info version 7.2 and SPSS version 24 were used for data entry and analysis respectively. Binary and multivariable logistic regression analyses were used to characterize the association between dependent and independent variables.

Results: Among 383 respondents $45.6 \%$ and $35.8 \%$ reported to have positive attitude and good practice respectively. Receiving training on infection prevention and getting vaccinated against hepatitis $B$ are significantly associated with nurse's attitude. Nurses who served for more than ten years and those vaccinated against hepatitis $B$ are also found to have good practice.

Conclusion: The overall level of nurse's attitude and practice towards patients with hepatitis $B$ and $C$ viruses is significantly low. Therefor nurses need to get training on infection prevention and hepatitis $B$ vaccine for free. Proper supply of personal protective equipment should also be assured.

\section{Background}

Viral hepatitis is a leading cause of morbidity and mortality from liver diseases worldwide. About 1.34 million deaths from viral hepatitis were reported globally by the year 2015. Hepatitis B virus (HBV) and hepatitis $C$ virus (HCV) accounted for $96 \%$ of viral hepatitis related mortality. In 2015 WHO estimated 257 million and 71 million global cases of HBV and HCV respectively. The majority of these cases were in Asia and Africa (1). A systemic review and meta-analysis on hepatitis viruses in Ethiopia showed $7.4 \%$ (95\% Cl: $6.5-8.4)$ pooled prevalence of HBV and 3.1\% (95\% Cl: 2.2-4.4 for that of anti-hepatitis C virus antibody (2).

Hepatitis viruses $B$ and $C$ presents at a higher concentration in infected blood. These viruses are also detected in exudates, semen, and vaginal secretion and to the lowest amount in saliva $(3,4)$. Easy transmission from infected blood and body fluid (BBF) puts them as major occupational hazards for health care professionals especially for those who spend much time with these patients. Nurses are among the professionals spending much time with patients and reporting the highest proportion of exposure to $\operatorname{BBF}(5,6)$. 
Following infection prevention techniques is the basis for avoiding HBV and HCV transmission in health care setting. Additionally, individuals exposed for BBF or needle stick injury should wash the area with soap and water and get hepatitis B immune globulin (HBIG) as a prophylaxis Vaccination against hepatitis $B$ also helps to protect self from acquiring the virus. However, there is no effective vaccine against $\operatorname{HCV}(7,8)$.

In 2016 WHO approved global strategy to eliminate viral hepatitis as a global public health treat by the year 2030 (9). Action plans has been established in each WHO regions for the accomplishment of this global strategy. In Africa, regional action plan is formulated for 2016 to 2020 targeting at preventing new $\mathrm{HBV}$ and $\mathrm{HCV}$ infection by $30 \%$, reducing $\mathrm{HBV}$ and $\mathrm{HCV}$ related death by $10 \%$, delivering high quality care and stopping stigma and discrimination of patients (10). However, viral hepatitis prevention has faced many challenges in countries with limited resources. Low community awareness, lack of screening services and timely care are among the challenges to eliminate viral hepatitis especially hepatitis viruses $B$ and $C(11)$.

Patients with hepatitis B and C are subjected to stigmatization from friends, family members as well as health care workers. Fear of stigmatization also causes many patients not to disclose their condition for their close families. This in turn affects their quality of life and increase disease transmission and progression (12).

The stigma and discrimination are even worse when nurses spending much time with these patients practice it. Infectiousness of the viruses and lack of confidence in protecting self are the reasons for nurses to face an ethical dilemma in deciding whether to care with dignity and equality for HBV and HCV infected individuals. Previous studies showed that some nurses feel anxious about acquiring HBV and $\mathrm{HCV}$, while others feel confident to protect them self when caring for these patients (13).

Research studies reveal that nurses had better knowledge of HBV and HCV but fail to comply with preventive practices against HBV and HCV. This increases nurse's exposure to HBV and HCV and compromises the quality of care given for these patients. Better knowledge, positive attitude and good practice towards caring for patients with hepatitis $\mathrm{B}$ and $\mathrm{C}$ are vital in properly treating as well as preventing transmission of hepatitis viruses $B$ and $C(14,15)$.

Nevertheless, studies done in Ethiopia are not sufficient enough to address attitude and practice towards $\mathrm{HBV}$ and HCV infected individuals. So that the aim of this study was to assess attitude and practice of nurses towards hepatitis $\mathrm{B}$ and $\mathrm{C}$ patients and to provide recommendations based on study findings.

\section{Methods}

\section{Study design and setting}

Institution based cross sectional study design was implemented from April 01 to 30, 2019 at governmental hospitals in Addis Ababa. Addis Ababa is the capital city of Ethiopia where the African 
union is headquartered. The city has 51 hospitals of which 14 are governmental. Nurses participated in this study were selected from five randomly selected governmental hospitals namely Tikur Anbesa Specialized Hospital (TASH), Alert hospital, Zewditu memorial hospital, Yekatit 12 hospital and Minilik II hospital.

\section{Study population and recruitment criteria}

Nurses who had at least one-year clinical experience and selected randomly from selected governmental hospitals in Addis Ababa were participated in the study.

\section{Sample size determination and sampling procedure}

The sample size was determined using single population proportion formula. Based on the assumption $5 \%$ margin of error, $95 \%$ confidence interval $(\mathrm{Cl})$ and taking $42.6 \%$ good practice from a study done in Jimma (16), the actual sample size for the study was calculated as follows.

$$
\mathrm{ni}=\left(\underline{\mathrm{Z}}_{\underline{\alpha} 2}\right)^{2} \mathrm{pq}
$$

$d^{2}$

Where $\mathrm{ni}=$ the minimum sample size required

$Z_{\mathrm{a} / 2}=$ standard normal deviation, set at 1.96 , to correspond to the $95 \%$ confidence interval

$P=0.43$

$q=1.0-p$

$d=$ margin of error/an absolute precision $=5 \%=0.05$

$$
\mathrm{ni}=(1.96)^{2}(0.43)(1-0.43)=377
$$

$(0.05)^{2}$

By adding $5 \%$ for non-responses the final sample size became 396 .

Five governmental hospitals in Addis Ababa (TASH, Alert hospital, Zewditu memorial hospital, Menlik II hospital and Yekatit 12 hospital) were selected using simple random sampling technique. To determine the number of nurses to be participated in study from each of the selected hospitals proportional allocation was applied. Finally, study participants from each selected hospital were selected by simple random sampling technique.

\section{Study variables}

Dependent variables: Attitude and practice of nurses towards patients with hepatitis $B$ and $C$ viruses 
Independent variables: age, sex, marital status, years of work experience, training on infection prevention, history of exposure to BBF and needle and sharp injuries from HBV and HCV infected patients, vaccination against $\mathrm{HBV}$ and attitude towards patients with $\mathrm{HBV}$ and $\mathrm{HCV}$

\section{Data collection tool and techniques}

Data was collected from the study participants using semi structured self-administered questionnaire. The tool was adapted from published articles $(14,16-18)$. Internal consistency of the tool was checked by Cronbach's alpha which was 0.75 for attitude items, 0.73 for practice items and 0.79 for both.

\section{Operational definitions}

Attitude: intention and acceptance towards care of patients with hepatitis B and C viruses. Likert's fivepoint scale was used in rating of attitude related items and were scored 1 to 5 (1-strongly disagree, 2disagree, 3-neutral, 4-agree, 5-strongly agree for positive statements and the reverse for negative ones). The scores for each statement were added to give maximum of 45 and minimum of 9 . Positive attitude was given for interviewee who scored 32 and above whereas; negative attitude was given for interviewee who scored below 32 (19).

Practice: Nurses way of care for patients with hepatitis B and C viruses. Rating of practice related items was from 0-4 (0-never, 1-seldom, 2-sometimes, 3-usually, 4-always for recommended practices and the reverse for non-recommended practices). The scores for each item were added giving maximum of 40 and minimum of 0 . Nurses scored 28 and above were considered to have good practice and those who scored $<28$ were taken to have poor practice (19).

\section{Data processing and analysis}

The data was coded, cleaned, and entered into Epi info version 7.2 and then exported to SPSS window version 24 for analysis. Descriptive statistics, frequency, and proportion were computed. The association between each independent variable and the outcome variables was assessed by using binary logistic regression. All variables with $\mathrm{P} \leq 0.25$ in the binary logistic regression analysis were further taken to multivariable logistic regression analysis in order to control all possible confounders. Adjusted odds ratio along with $95 \% \mathrm{Cl}$ and P-value $<0.05$ were considered to declare factors that have statistically significant association with nurse's attitude and practice towards patients' hepatitis $B$ and $C$ viruses.

\section{Results}

\section{Socio-demographic characteristics of nurses}

From the total of 396 study participants 383 respond to the questionnaire which gives response rate of $96.7 \%$. About two third $(67.1 \%)$ of the respondents were females and more than half $(52.5 \%)$ in the age group of $22-29$ with mean age of 31.4 . Nearly half (51.7\%) were single, $173(45.2 \%)$ had monthly income 
of 3500 to 5000 ETB, 334 (87.2\%) were BSC nurses, 90.3\% staff and 9.7\% head nurses, 189 (49.3\%) worked for 1 to 5 years in health care. (Table:1)

Table 1: Socio-demographic characteristics of nurses working in governmental hospitals in AA, Ethiopia, $2019(\mathrm{~N}=383)$ 


\begin{tabular}{|c|c|c|c|}
\hline Characteristics & Category & Number & Percent \\
\hline \multirow[t]{2}{*}{ Sex } & Male & 126 & 32.9 \\
\hline & Female & 257 & 67.1 \\
\hline \multirow[t]{3}{*}{ Age $(\min =22, \max =56$, mean $=31.4, S D=7.52)$} & $22-29$ & 201 & 52.5 \\
\hline & $30-39$ & 132 & 34.5 \\
\hline & $>40$ & 50 & 13.1 \\
\hline \multirow[t]{4}{*}{ Marital Status } & Single & 198 & 51.7 \\
\hline & Married & 172 & 44.9 \\
\hline & Divorced & 8 & 2.1 \\
\hline & Widowed & 5 & 1.3 \\
\hline \multirow[t]{3}{*}{ Monthly income } & $<3500$ ETB & 52 & 13.6 \\
\hline & 3500-5000ETB & 173 & 45.2 \\
\hline & $>5000 \mathrm{ETB}$ & 158 & 41.3 \\
\hline \multirow[t]{3}{*}{ Educational level } & Diploma & 33 & 8.6 \\
\hline & BSC & 334 & 87.2 \\
\hline & MSC & 16 & 4.2 \\
\hline \multirow[t]{5}{*}{ Working hospital } & TASH & 151 & 39.4 \\
\hline & Yekatit 12 & 70 & 18.3 \\
\hline & Alert & 63 & 16.4 \\
\hline & Menelik II & 51 & 13.3 \\
\hline & Zewditu & 48 & 12.5 \\
\hline \multirow[t]{8}{*}{ Working unit } & Emergency & 54 & 14.1 \\
\hline & OPD & 82 & 21.4 \\
\hline & Pediatrics ward & 26 & 6,8 \\
\hline & Medical wards & 66 & 17.2 \\
\hline & Surgical wards & 54 & 14.1 \\
\hline & Orthopedics ward & 11 & 2.9 \\
\hline & ICUs & 31 & 8.1 \\
\hline & OR & 48 & 12.5 \\
\hline
\end{tabular}




\begin{tabular}{|llll|} 
& Others & 11 & 2.9 \\
\hline Work experience $(\min =1$, & $1-5$ years & 189 & 49.3 \\
\hline Max=34, Mean=7.58, SD=7.09) & 6-10 years & 108 & 28.2 \\
\cline { 2 - 3 } & $>1$ 10years & 8622.5 & \\
\hline
\end{tabular}

\section{Nurses attitude towards patients with hepatitis B and C Viruses}

About $176(46 \%)$ of the respondents disagree or strongly disagree to be reluctant to take care of patients with hepatitis B and C viruses. Sixty-six-point one percent of them disagree or strongly disagree to the statement I am afraid of going near patients infected with hepatitis B and C. Regarding fear of getting infection from these viruses 164 (42.8\%) disagreed, 112 (29.2\%) strongly disagreed and only $5.7 \%$ strongly agreed. About $163(42.4 \%)$ of the respondents agreed and $76(19.8 \%)$ strongly agreed that they are confident to treat hepatitis B and C patients effectively and safely. Infection control guidelines are believed by majority $(85.4 \%)$ of the respondents to protect self against these viruses. In general, less than half $(45.6 \%)$ of the respondents were found to have positive attitude towards patients with hepatitis B and $\mathrm{C}$ viruses. (Table: 2 )

Table 2: Attitude towards patients with hepatitis $B$ and $C$ viruses of nurses working in governmental hospitals in AA, Ethiopia, 2019 ( $N=383)$. 


\begin{tabular}{|c|c|c|c|c|c|}
\hline \multirow[t]{4}{*}{ Variable } & \multicolumn{5}{|l|}{ Category } \\
\hline & $\begin{array}{l}\text { Strongly } \\
\text { agree }\end{array}$ & Agree & Neutral & Disagree & $\begin{array}{l}\text { Strongly } \\
\text { disagree }\end{array}$ \\
\hline & № & № & № & № & № \\
\hline & $\%$ & $\%$ & $\%$ & $\%$ & $\%$ \\
\hline \multirow{2}{*}{$\begin{array}{l}\text { I am reluctant to take care of patients with } \\
\text { hepatitis } B \text { and } C \text { viruses }\end{array}$} & 58 & 114 & 35 & 103 & 73 \\
\hline & 15.1 & 29.8 & 9.1 & 26.9 & 19.1 \\
\hline \multirow{2}{*}{$\begin{array}{l}\text { I am afraid of going near patients infected } \\
\text { with hepatitis } B \text { and } C \text { viruses }\end{array}$} & 26 & 73 & 31 & 134 & 119 \\
\hline & 6.8 & 19.1 & 8.1 & 35.0 & 31.1 \\
\hline \multirow{2}{*}{$\begin{array}{l}\text { I have no concern of being infected with } \\
\text { hepatitis } B \text { and } C \text { viruses }\end{array}$} & 22 & 50 & 35 & 164 & 112 \\
\hline & 5.7 & 13.1 & 9.1 & 42.8 & 29.2 \\
\hline \multirow{2}{*}{$\begin{array}{l}\text { I am confident that I have the skills needed to } \\
\text { effectively and safely treat patients with } \\
\text { hepatitis B and C viruses }\end{array}$} & 76 & 163 & 52 & 68 & 24 \\
\hline & 19.8 & 42.4 & 13.6 & 17.8 & 6.3 \\
\hline \multirow{2}{*}{$\begin{array}{l}\text { Following infection control guidelines } \\
\text { protects me from being infected with hepatitis } \\
B \text { and C viruses }\end{array}$} & 177 & 150 & 26 & 24 & 6 \\
\hline & 46.2 & 39.2 & 6.8 & 6.3 & 1.6 \\
\hline \multirow{2}{*}{$\begin{array}{l}\text { People with hepatitis } B \text { and } C \text { should be } \\
\text { treated in a separate room in a hospital }\end{array}$} & 61 & 114 & 36 & 115 & 57 \\
\hline & 15.9 & 29.8 & 9.4 & 30.0 & 14.9 \\
\hline \multirow{2}{*}{$\begin{array}{l}\text { Health professionals who are HBV or HCV } \\
\text { positive should be discouraged from having } \\
\text { contact with patients }\end{array}$} & 51 & 73 & 47 & 131 & 81 \\
\hline & 13.3 & 19.1 & 12.3 & 34.2 & 21.1 \\
\hline \multirow{2}{*}{$\begin{array}{l}\text { Relatives of patients with hepatitis B and C } \\
\text { should be notified of the patient's status even } \\
\text { without patients consent }\end{array}$} & 64 & 97 & 52 & 111 & 59 \\
\hline & 16.7 & 25.3 & 13.6 & 29.0 & 15.4 \\
\hline \multirow{2}{*}{$\begin{array}{l}\text { Patients with hepatitis } B \text { and } C \text { viruses should } \\
\text { not be appointed for follow up care }\end{array}$} & 24 & 48 & 36 & 151 & 124 \\
\hline & 6.3 & 12.5 & 9.4 & 39.4 & 32.4 \\
\hline
\end{tabular}

\section{Nurses practice towards patients with hepatitis $B$ and $C$ viruses}

Majority (82.5\%) of the respondents always use gloves for hepatitis B and C patient contact and $71.8 \%$ of the respondents always change gloves after having contact to these patients. About three fourth (76.2\%) of nurses always dispose sharp and needles used for hepatitis B and C patients properly. Nearly half $(49.1 \%)$ of the respondents always use additional infection control measures while, $27.7 \%$ always double gloves for hepatitis B and C patient care that they didn't use for other patients. More than half $(52.0 \%)$ of 
the respondents always mark hepatitis $B$ and $C$ status on the chart. In this study, it is also found as only $110(28.7 \%)$ always deliver the same standard of care for hepatitis B and $C$ patients as they did for others. In general, nearly two third (64.2\%) of participants has reported poor practice towards care of patients with hepatitis B and C. (Table: 3 )

Table 3: Practice towards patients with hepatitis $B$ and $C$ viruses of nurses working in governmental hospitals in AA, Ethiopia, 2019 ( $N=383)$. 


\begin{tabular}{|c|c|c|c|c|c|}
\hline \multirow[t]{4}{*}{ Variable } & \multicolumn{5}{|c|}{ Category } \\
\hline & Always & Usually & Sometimes & Seldom & Never \\
\hline & № & №. & №. & №. & №. \\
\hline & $\%$ & $\%$ & $\%$ & $\%$ & $\%$ \\
\hline \multirow{2}{*}{$\begin{array}{l}\text { Using gloves for hepatitis B and C patient } \\
\text { contact }\end{array}$} & 316 & 41 & 19 & 4 & 3 \\
\hline & 82.5 & 10.7 & 5.0 & 1.0 & 0.8 \\
\hline \multirow{2}{*}{$\begin{array}{l}\text { Changing gloves after contact with hepatitis } \\
B \text { and } C \text { viruses infected patients }\end{array}$} & 275 & 57 & 38 & 11 & 2 \\
\hline & 71.8 & 14.9 & 9.9 & 2.9 & 0.5 \\
\hline \multirow{2}{*}{$\begin{array}{l}\text { Disposing needle/ sharps used for hepatitis } \\
B \text { and C patients properly }\end{array}$} & 292 & 56 & 26 & 4 & 5 \\
\hline & 76.2 & 14.6 & 6.8 & 1.0 & 1.3 \\
\hline \multirow{2}{*}{$\begin{array}{l}\text { Using additional infection control measures } \\
\text { while caring for hepatitis B and C patients } \\
\text { that you don't use for other patients }\end{array}$} & 188 & 96 & 77 & 12 & 10 \\
\hline & 49.1 & 25.1 & 20.1 & 3.1 & 2.6 \\
\hline \multirow{2}{*}{$\begin{array}{l}\text { Wearing double gloves for contact with } \\
\text { hepatitis B and C patients }\end{array}$} & 106 & 68 & 121 & 35 & 53 \\
\hline & 27.7 & 17.8 & 31.6 & 9.1 & 13.8 \\
\hline \multirow[t]{2}{*}{ Marking hepatitis $B$ and $C$ status on the chart } & 199 & 80 & 69 & 9 & 26 \\
\hline & 52.0 & 20.9 & 18.0 & 2.3 & 6.8 \\
\hline \multirow{2}{*}{$\begin{array}{l}\text { Avoiding physical contact with hepatitis B } \\
\text { and C patients }\end{array}$} & 7 & 64 & 68 & 81 & 163 \\
\hline & 1.8 & 16.7 & 17.8 & 21.1 & 42.6 \\
\hline \multirow{2}{*}{$\begin{array}{l}\text { Delivering the same standard of care to } \\
\text { patients with hepatitis } B \text { and } C \text { viruses as } \\
\text { you do for other patients }\end{array}$} & 110 & 111 & 97 & 26 & 39 \\
\hline & 28.7 & 29.0 & 25.3 & 6.8 & 10.2 \\
\hline \multirow{2}{*}{$\begin{array}{l}\text { Responding to hepatitis B and C patients' } \\
\text { needs timely }\end{array}$} & 149 & 118 & 89 & 15 & 12 \\
\hline & 38.9 & 30.8 & 23.2 & 3.9 & 3.1 \\
\hline \multirow{2}{*}{$\begin{array}{l}\text { Talking positively about patients living with } \\
\text { hepatitis } B \text { and } C\end{array}$} & 147 & 88 & 60 & 38 & 50 \\
\hline & 38.4 & 23.0 & 15.7 & 9.9 & 13.1 \\
\hline
\end{tabular}

\section{Occupational exposure and vaccination status}

Regarding to occupational exposure $43(11.2 \%)$ of the respondents had history of exposure for BBF from HBV and HCV infected patient while, 19 (5.0\%) ever sustained needle stick injury from such patients.

Washing with soap and water was the major action taken after BBF and needle stick injury done by $74.4 \%$ 
and $73.7 \%$ of nurses respectively. About three fourth $(73.9 \%)$ of the respondents were fully vaccinated for hepatitis B while 317 (82.8\%) took two doses and 333

$(86.9 \%)$ took at least one dose of hepatitis B vaccine. Thirteen-point one percent of them didn't take hepatitis $B$ vaccine at all.

Factors associated with the level of nurse's attitude and practice towards patients with hepatitis $B$ and $C$ viruses

Multivariable regression analysis for nurse's level of attitude with independent variables showed nurses who attended training on infection prevention to be 2.1times more positive for hepatitis $B$ and $C$ patients than who didn't get training on infection prevention [AOR: 2.1; $95 \mathrm{Cl}$ (1.33-3.42)]. Getting hepatitis B vaccination also found to increase the likely to have positive attitude by 6.6 [AOR: $6.6 ; 95 \% \mathrm{Cl}(2.78$ 17.28)] (Table 4).

Table 4: Regression analysis of attitude towards patients with hepatitis $B$ and $C$ among nurses working in public hospitals in Addis Ababa, Ethiopia, 2019 ( $N=383)$. 


\begin{tabular}{|c|c|c|c|c|c|c|c|}
\hline \multirow[t]{2}{*}{ Variables } & \multirow[t]{2}{*}{ Category } & \multicolumn{2}{|c|}{ Level of attitude } & \multicolumn{2}{|c|}{ Odd ratio at $95 \% \mathrm{Cl}$ ) } & \multirow[b]{2}{*}{$\begin{array}{l}\text { Adjusted } \\
\text { odds ratio }\end{array}$} & \multirow[b]{2}{*}{$\begin{array}{l}\mathrm{p}- \\
\text { value }\end{array}$} \\
\hline & & Positive & Negative & $\begin{array}{l}\text { Crude } \\
\text { odds ratio }\end{array}$ & $\begin{array}{l}\mathrm{p}- \\
\text { value }\end{array}$ & & \\
\hline \multirow[t]{2}{*}{ Sex } & Male & $\begin{array}{l}66 \\
(52.4)\end{array}$ & $\begin{array}{l}60 \\
(47.6)\end{array}$ & $\begin{array}{l}1.52(0.99- \\
2.3)\end{array}$ & 0.056 & & \\
\hline & Female & $\begin{array}{l}108 \\
(42.0)\end{array}$ & $\begin{array}{l}149 \\
(58.0)\end{array}$ & 1 & & & \\
\hline \multirow[t]{3}{*}{ Age } & $22-29$ & $\begin{array}{l}84 \\
(41.8)\end{array}$ & $\begin{array}{l}117 \\
(58.2)\end{array}$ & 1 & & & \\
\hline & $30-39$ & $\begin{array}{l}60 \\
(45.5)\end{array}$ & $\begin{array}{l}72 \\
(54.5)\end{array}$ & $\begin{array}{l}1.16(0.75- \\
1.81)\end{array}$ & 0.509 & & \\
\hline & $\geq 40$ & $\begin{array}{l}30 \\
(60.0)\end{array}$ & $\begin{array}{l}20 \\
(40.0)\end{array}$ & $\begin{array}{l}2.09(1.11- \\
3.93)\end{array}$ & 0.022 & & \\
\hline \multirow[t]{4}{*}{ Marital status } & Single & $84(42.4$ & $\begin{array}{l}114 \\
(57.6)\end{array}$ & 1 & & & \\
\hline & Married & $\begin{array}{l}81 \\
(47.1)\end{array}$ & $\begin{array}{l}91 \\
(52.9)\end{array}$ & $\begin{array}{l}1.21(0.08- \\
1.82)\end{array}$ & 0.368 & & \\
\hline & Divorced & $6(75.0)$ & $2(25.0)$ & $\begin{array}{l}4.07(0.08- \\
20.68)\end{array}$ & 0.090 & & \\
\hline & Widowed & $3(60.0)$ & $2(40.0)$ & $\begin{array}{l}2.04(0.33- \\
12.45)\end{array}$ & 0.442 & & \\
\hline \multirow[t]{3}{*}{ Monthly income } & $<3500$ ETB & $\begin{array}{l}19 \\
(36.5)\end{array}$ & $\begin{array}{l}33 \\
(63.5)\end{array}$ & 1 & & & \\
\hline & $\begin{array}{l}3500- \\
5000 \mathrm{ETB}\end{array}$ & $\begin{array}{l}68 \\
(39.3)\end{array}$ & $\begin{array}{l}105 \\
(60.7)\end{array}$ & $\begin{array}{l}1.13(0.59- \\
2.14)\end{array}$ & 0.719 & & \\
\hline & $>5000 \mathrm{ETB}$ & $\begin{array}{l}87 \\
(55.1)\end{array}$ & $\begin{array}{l}71 \\
(44.9)\end{array}$ & $\begin{array}{l}2.13(1.12- \\
4.04)\end{array}$ & 0.022 & & \\
\hline \multirow[t]{3}{*}{ Educational level } & Diploma & $\begin{array}{l}11 \\
(33.3)\end{array}$ & $\begin{array}{l}22 \\
(66.6)\end{array}$ & 1 & & & \\
\hline & BSC & $\begin{array}{l}151 \\
(45.2)\end{array}$ & $\begin{array}{l}183 \\
(54.8)\end{array}$ & $\begin{array}{l}1.65(0.77- \\
3.51)\end{array}$ & 0.194 & & \\
\hline & MSC & $\begin{array}{l}12 \\
(75.0)\end{array}$ & $4(25.0)$ & $\begin{array}{l}6.00(1.57- \\
22.99)\end{array}$ & 0.009 & & \\
\hline \multirow[t]{2}{*}{ Position } & Staff & $\begin{array}{l}147 \\
(42.4)\end{array}$ & $\begin{array}{l}199 \\
(57.5)\end{array}$ & 1 & & & \\
\hline & Head & $\begin{array}{l}27 \\
(73.0)\end{array}$ & $\begin{array}{l}10 \\
(27.0)\end{array}$ & $\begin{array}{l}3.66(1.72- \\
7.79)\end{array}$ & 0.001 & & \\
\hline Working unit & Emergency & $\begin{array}{l}21 \\
(38.9)\end{array}$ & $\begin{array}{l}33 \\
(61.1)\end{array}$ & $\begin{array}{l}1.70(0.40- \\
7.13)\end{array}$ & 0.470 & & \\
\hline
\end{tabular}




\begin{tabular}{|c|c|c|c|c|c|c|c|}
\hline & OPD & $\begin{array}{l}47 \\
(57.3)\end{array}$ & $\begin{array}{l}35 \\
(42.7)\end{array}$ & $\begin{array}{l}3.58(0.89- \\
14.48)\end{array}$ & \multicolumn{2}{|l|}{0.074} & \\
\hline & $\begin{array}{l}\text { Pediatrics } \\
\text { ward }\end{array}$ & $\begin{array}{l}12 \\
(46.2)\end{array}$ & $\begin{array}{l}14 \\
(53.8)\end{array}$ & $\begin{array}{l}2.29(0.49- \\
10.61)\end{array}$ & \multicolumn{2}{|l|}{0.291} & \\
\hline & $\begin{array}{l}\text { Medical } \\
\text { ward }\end{array}$ & 21(31.8) & $\begin{array}{l}45 \\
(68.2)\end{array}$ & $\begin{array}{l}1.24(0.30- \\
5.17)\end{array}$ & \multicolumn{2}{|l|}{0.763} & \\
\hline & $\begin{array}{l}\text { surgical } \\
\text { ward }\end{array}$ & $\begin{array}{l}25 \\
(46.3)\end{array}$ & $\begin{array}{l}29 \\
(53.7)\end{array}$ & $\begin{array}{l}2.30(0.55- \\
9.61)\end{array}$ & \multicolumn{2}{|l|}{0.254} & \\
\hline & $\begin{array}{l}\text { Orthopedic } \\
\text { ward }\end{array}$ & $3(27.3)$ & $8(72.7)$ & 1 & & & \\
\hline & ICUs & $\begin{array}{l}15 \\
(48.4)\end{array}$ & $\begin{array}{l}16 \\
(51.6)\end{array}$ & $\begin{array}{l}2.50(0.56- \\
11.23)\end{array}$ & \multicolumn{2}{|l|}{0.232} & \\
\hline & OR & $\begin{array}{l}24 \\
(50.0)\end{array}$ & $\begin{array}{l}24 \\
(50.0)\end{array}$ & $\begin{array}{l}2.67(0.63- \\
11.28)\end{array}$ & \multicolumn{2}{|l|}{0.183} & \\
\hline & Others & $6(54.5)$ & $5(45.5)$ & $\begin{array}{l}3.200 \\
(0.54- \\
18.98)\end{array}$ & \multicolumn{3}{|l|}{0.200} \\
\hline \multirow[t]{3}{*}{ Work experience } & $1-5$ & $\begin{array}{l}82 \\
(43.4)\end{array}$ & $\begin{array}{l}107 \\
(56.6)\end{array}$ & $\begin{array}{l}1.20(0.74- \\
1.95)\end{array}$ & \multicolumn{2}{|l|}{0.450} & \\
\hline & $6-10$ & $\begin{array}{l}42 \\
(38.9)\end{array}$ & $\begin{array}{l}66 \\
(61.1)\end{array}$ & 1 & & & \\
\hline & $>10$ & $\begin{array}{l}50 \\
(58.1)\end{array}$ & $\begin{array}{l}36 \\
(41.9)\end{array}$ & $\begin{array}{l}2.18(1.23- \\
3.89)\end{array}$ & 0.008 & & \\
\hline \multirow[t]{2}{*}{ Training on IP } & Yes & $\begin{array}{l}93 \\
(57.8)\end{array}$ & $\begin{array}{l}68 \\
(42.2)\end{array}$ & $\begin{array}{l}2.38(1.57- \\
3.60)\end{array}$ & 0.000 & $\begin{array}{l}2.13(1.33- \\
3.42)\end{array}$ & \multirow[t]{2}{*}{0.002} \\
\hline & No & $\begin{array}{l}81 \\
(36.5)\end{array}$ & $\begin{array}{l}141 \\
(63.5)\end{array}$ & 1 & & & \\
\hline \multirow[t]{2}{*}{ Exposure to BBF } & Yes & $\begin{array}{l}19 \\
(44.2)\end{array}$ & $\begin{array}{l}24 \\
(55.8)\end{array}$ & 1 & & & \\
\hline & No & $\begin{array}{l}155 \\
(45.6)\end{array}$ & $\begin{array}{l}185 \\
(54.4)\end{array}$ & $\begin{array}{l}1.06(0.56- \\
2.00)\end{array}$ & 0.862 & & \\
\hline \multirow[t]{2}{*}{$\begin{array}{l}\text { History of needle } \\
\text { stick injury }\end{array}$} & Yes & $\begin{array}{l}11 \\
(57.9)\end{array}$ & $8(42.1)$ & $\begin{array}{l}1.70(0.67- \\
4.31)\end{array}$ & \multicolumn{2}{|l|}{0.268} & \\
\hline & No & $\begin{array}{l}163 \\
(44.8)\end{array}$ & $\begin{array}{l}201 \\
(55.2)\end{array}$ & 1 & & & \\
\hline \multirow[t]{2}{*}{$\begin{array}{l}\text { Vaccination } \\
\text { against HBV }\end{array}$} & Yes & $\begin{array}{l}167 \\
(50.2)\end{array}$ & $\begin{array}{l}166 \\
(49.8)\end{array}$ & $\begin{array}{l}6.18(2.70- \\
14.14)\end{array}$ & 0.000 & $\begin{array}{l}6.63 \\
(2.78- \\
17.26)\end{array}$ & \multirow[t]{2}{*}{0.000} \\
\hline & No & $7(14.0)$ & $\begin{array}{l}43 \\
(86.0)\end{array}$ & 1 & & & \\
\hline
\end{tabular}


Regarding with factors affecting practice towards patients with hepatitis $B$ and $C$ viruses nurses served for more than 10 years were 3.8 times more likely to have good practice than those who worked for 1 to 5 years [AOR: $3.8 ; 95 \% \mathrm{Cl}(1.31-10.98)]$. Nurses who sustained needle stick injury from hepatitis $\mathrm{B}$ and $\mathrm{C}$ patients had 5.2 times likely of having poor practice than those who didn't sustained injury [AOR: 5.2; $95 \% \mathrm{Cl}(1.24-21.86)]$. Vaccination against hepatitis $\mathrm{B}$ and positive attitude were also found to have significant association with nurse's practice towards hepatitis $B$ and $C$ patients with $P$-values of 0.006 and 0.000 respectively (Table 5).

Table 5: Regression analysis of practice towards patients with hepatitis $B$ and $C$ among nurses working in public hospitals in Addis Ababa, Ethiopia, 2019 ( $N=383)$. 


\begin{tabular}{|c|c|c|c|c|c|c|c|}
\hline \multirow[t]{2}{*}{ Variables } & \multirow[t]{2}{*}{ Category } & \multicolumn{2}{|c|}{ Level of practice } & \multicolumn{4}{|c|}{ Odd ratio $(95 \% \mathrm{Cl})$} \\
\hline & & Good & Poor & $\begin{array}{l}\text { Crude } \\
\text { odds ratio }\end{array}$ & $\begin{array}{l}\mathrm{p}- \\
\text { value }\end{array}$ & $\begin{array}{l}\text { Adjusted } \\
\text { odds ratio }\end{array}$ & $\begin{array}{l}p- \\
\text { value }\end{array}$ \\
\hline \multirow[t]{2}{*}{ Sex } & Male & $51(40.5)$ & 75 (59.5) & $\begin{array}{l}1.35 \\
(0.87- \\
2.10)\end{array}$ & 0.179 & & \\
\hline & Female & $86(33.5)$ & $\begin{array}{l}171 \\
(66.5)\end{array}$ & 1 & & & \\
\hline \multirow[t]{3}{*}{ Age } & $22-29$ & 60 (29.9) & $141(70.1)$ & 1 & & & \\
\hline & $30-39$ & $45(34.1))$ & $87(65.9)$ & $\begin{array}{l}1.22(0.71- \\
1.95)\end{array}$ & 0.416 & & \\
\hline & $\geq 40$ & $32(64.0)$ & $18(36.0)$ & $\begin{array}{l}4.18 \\
(2.18- \\
8.02)\end{array}$ & 0.000 & & \\
\hline \multirow[t]{4}{*}{ Marital status } & Single & $65(32.8)$ & $\begin{array}{l}133 \\
(67.2)\end{array}$ & 1 & & & \\
\hline & Married & $65(37.8)$ & $\begin{array}{l}107 \\
(62.2)\end{array}$ & $\begin{array}{l}1.24 \\
(0.81- \\
1.91)\end{array}$ & 0.319 & & \\
\hline & Divorced & $5(62.5)$ & $3(37.5)$ & $\begin{array}{l}3.41 \\
(0.79- \\
14.71)\end{array}$ & 0.100 & & \\
\hline & Widowed & $2(40.0)$ & $3(60.0)$ & $\begin{array}{l}1.364 \\
(0.22- \\
8.37)\end{array}$ & 0.737 & & \\
\hline \multirow{3}{*}{$\begin{array}{l}\text { Monthly } \\
\text { income }\end{array}$} & $<3500$ ETB & $12(23.1)$ & $40(76.9)$ & 1 & & & \\
\hline & $\begin{array}{l}3500- \\
5000 \text { ETB }\end{array}$ & $50(28.9)$ & $\begin{array}{l}123 \\
(71.1)\end{array}$ & $\begin{array}{l}1.36(0.66- \\
2.80)\end{array}$ & 0.411 & & \\
\hline & $>5000$ ETB & $75(47.5)$ & $83(52.5)$ & $\begin{array}{l}3.01(1.47- \\
6.17)\end{array}$ & 0.003 & & \\
\hline \multirow{3}{*}{$\begin{array}{l}\text { Educational } \\
\text { level }\end{array}$} & Diploma & $8(24.2)$ & $25(75.8)$ & 1 & & & \\
\hline & BSC & $121(36.2)$ & $\begin{array}{l}213 \\
(63.8)\end{array}$ & $\begin{array}{l}1.78(0.78- \\
4.06)\end{array}$ & 0.174 & & \\
\hline & MSC & $8(50.0)$ & $8(50.0)$ & $\begin{array}{l}5.13 \\
(0.88- \\
11.05)\end{array}$ & 0.007 & & \\
\hline \multirow[t]{2}{*}{ Position } & Staff & 115 (33.2) & $\begin{array}{l}231 \\
(66.8)\end{array}$ & 1 & & & \\
\hline & Head & $22(59.5)$ & $15(40.5)$ & $\begin{array}{l}2.95 \\
(1.47-\end{array}$ & 0.002 & & \\
\hline
\end{tabular}


5.89)

\begin{tabular}{|c|c|c|c|c|c|}
\hline \multirow[t]{9}{*}{ Working unit } & Emergency & 17 (31.5) & 37 (68.5) & $\begin{array}{l}3.24 \\
(1.58- \\
6.66)\end{array}$ & 0.001 \\
\hline & OPD & $40(48.8)$ & $42(51.2)$ & $\begin{array}{l}2.91 \\
(1.11- \\
7.63)\end{array}$ & 0.029 \\
\hline & $\begin{array}{l}\text { Pediatrics } \\
\text { ward }\end{array}$ & $12(46.2)$ & $14(53.8)$ & $\begin{array}{l}1.56 \\
(0.69- \\
3.52)\end{array}$ & 0.282 \\
\hline & $\begin{array}{l}\text { Medical } \\
\text { ward }\end{array}$ & 15 (22.7) & 51 (77.3) & 1 & \\
\hline & $\begin{array}{l}\text { surgical } \\
\text { ward }\end{array}$ & 15 (27.8) & 39 (72.2) & $\begin{array}{l}1.31 \\
(0.51- \\
2.99)\end{array}$ & 0.526 \\
\hline & $\begin{array}{l}\text { Orthopedics } \\
\text { ward }\end{array}$ & $4(36.4)$ & $7(63.6)$ & $\begin{array}{l}1.94 \\
(0.50- \\
7.55)\end{array}$ & 0.337 \\
\hline & ICUs & $14(45.2)$ & $17(54.8)$ & $\begin{array}{l}2.80 \\
(1.13- \\
6.97)\end{array}$ & 0.027 \\
\hline & OR & $17(35.4)$ & $31(64.6)$ & $\begin{array}{l}1.87 \\
(0.82- \\
4.26)\end{array}$ & 0.139 \\
\hline & Others & $3(27.3)$ & $8(72.7)$ & $\begin{array}{l}1.28 \\
(0.30- \\
5.42)\end{array}$ & 0.742 \\
\hline
\end{tabular}

\begin{tabular}{|c|c|c|c|c|c|c|c|}
\hline \multirow[t]{3}{*}{$\begin{array}{l}\text { Work } \\
\text { experience }\end{array}$} & $1-5$ & 59 (31.2) & $\begin{array}{l}130 \\
(68.8)\end{array}$ & 1 & & & \multirow{3}{*}{0.014} \\
\hline & $6-10$ & $27(25.0)$ & $81(75.0)$ & $\begin{array}{l}1.36 \\
(0.80- \\
2.32)\end{array}$ & 0.257 & & \\
\hline & $>10$ & 52 (59.3) & 35 (40.7) & $\begin{array}{l}4.37 \\
(2.37- \\
8.06)\end{array}$ & 0.000 & $\begin{array}{l}3.79 \\
(1.31- \\
10.97)\end{array}$ & \\
\hline
\end{tabular}

$\begin{array}{lllll}\text { Training on IP } \quad \text { Yes } \quad 75(46.6) & 86(53.4) & 2.25 & 0.000 \\ & & & (1.47- & \\ & & 3.48) & \end{array}$

$\begin{array}{llll}\text { No } & 62(27.9) & 160 & 1 \\ & & (72.1) & \end{array}$

\begin{tabular}{|c|c|c|c|c|}
\hline $\begin{array}{l}\text { Exposure to } \\
\text { BBF }\end{array}$ & Yes & $16(37.2)$ & $27(62.8)$ & $\begin{array}{l}1.07 \\
(0.56- \\
2.07)\end{array}$ \\
\hline
\end{tabular}




\begin{tabular}{|c|c|c|c|c|c|c|c|}
\hline & No & $121(35.6)$ & $\begin{array}{l}219 \\
(64.4)\end{array}$ & 1 & & & \\
\hline \multirow{2}{*}{$\begin{array}{l}\text { History of } \\
\text { needle stick } \\
\text { injury }\end{array}$} & Yes & $4(21.1)$ & $15(78.9)$ & 1 & & & \multirow[b]{2}{*}{0.025} \\
\hline & No & $133(36.5)$ & $\begin{array}{l}231 \\
(63.5)\end{array}$ & $\begin{array}{l}2.16(0.70 \\
-6.64)\end{array}$ & 0.179 & $\begin{array}{l}5.20 \\
(1.24- \\
21.86)\end{array}$ & \\
\hline \multirow[t]{2}{*}{$\begin{array}{l}\text { Vaccination } \\
\text { against HBV }\end{array}$} & Yes & $131(39.3)$ & $\begin{array}{l}202 \\
(60.7)\end{array}$ & $\begin{array}{l}4.76 \\
(1.97- \\
11.48)\end{array}$ & 0.001 & $\begin{array}{l}4.50(1.56- \\
13.02)\end{array}$ & \multirow[t]{2}{*}{0.006} \\
\hline & No & $6(12.0)$ & $44(88.0)$ & 1 & & & \\
\hline & Positive & $100(57.5)$ & $74(42.5)$ & \multirow{2}{*}{$\begin{array}{l}6.28(3.95- \\
10.00)\end{array}$} & \multirow[t]{2}{*}{0.000} & \multirow{2}{*}{$\begin{array}{l}3.86(1.93- \\
7.72)\end{array}$} & \multirow[t]{2}{*}{0.000} \\
\hline $\begin{array}{l}\text { Nurses } \\
\text { Attitude }\end{array}$ & Negative & $37(17.7)$ & 172(82.3) & & & & \\
\hline
\end{tabular}

\section{Discussion}

The result of this study revealed as $46.0 \%$ of the respondents disagree or strongly disagreed with being reluctant to care for hepatitis $B$ and $C$ infected patients. This is higher when compared with studies done in Kutahya Western Turkey where 35.2\% were willing and in South East Brazil only 20.6\% of the participants were willing to care for hepatitis $B$ and $C$ viruses infected patients $(17,20)$. This difference may be due to the study participants being all health care workers in these studies. However, the finding in this study is much lower than the study from Vietnam that reported $73.3 \%$ of the respondents to be willing to care for hepatitis B/C patients. Besides, in a study from Japan only $18 \%$ of nurses agreed or somewhat agreed as they were unwilling to care for these patients $(13,18)$. The possible reason for this difference may be lack of preventive and therapeutic facilities in hospitals that imposes fear of acquiring these infections.

It is revealed from this study that $72.0 \%$ of the respondents concerned with getting infection from hepatitis B and C viruses. This study is in line with the study from North West Ethiopia that $77.2 \%$ disagreed with being non concerned with getting hepatitis B infection (21). Relatively lower proportion (54\%) of participants in a study from Japan had anxiety related the risk of acquiring hepatitis B and C infections. Moreover, quarter $(25.9 \%)$ of nurses in the present study afraid going near patients infected with HBV and HCV. Additionally, $65.8 \%$ in the Vietnam and $79 \%$ of participants in the Japan studies don't care of acquiring these infections from infected collogues. Again in the Japan study $88 \%$ of the participants disagree and strongly disagreed to avoid infected colleagues $(13,18,22)$. This discrepancy may be due to lack of personal protective equipment and nurse's attitude for colleagues may be different for other patients.

Some respondents in this study showed discriminatory behaviors that include feeling hepatitis B and C patients to be treated in a separate room (45.7\%), not to be appointed for follow up care (18.8\%) and infected health care professionals to be discouraged from having patient contact (32.4\%). Additionally, 
$42 \%$ of the respondents need hepatitis B and C patients' status to be disclosed to their families even without patient's permission. Comparable to this study a report from South East Brazil revealed as 21.1\% of HCWs participated in the study need hepatitis $\mathrm{B}$ and $\mathrm{C}$ patients to be given the last appointment for the day. In that study also $53.8 \%$ of the participants need all patients to be screened and infected individuals to be identified for safety. These behaviors of nurses may come from the higher perception of risk and fear of hepatitis B and C infections (20).

Regarding confidence in protecting self against hepatitis B and C $62.2 \%$ feel confident to treat these patients safely. This is relatively consistent with the study from Japan that found as $59 \%$ of nurses agree or somewhat agree that they were confident to protect them against these infections. However, relatively higher proportion (70\%) reported in the Vietnam study. This discrepancy may be resulted from the difference in socio-demographic characteristics of respondents $(13,18)$.

Regarding nurse's practice of infection control measures, majority (82.5\%) of the respondents reported as they always use gloves while caring for hepatitis B and C patients. Relatively lower (73.3\%) of the participants in a study in Bangladesh and $76.7 \%$ in Nigeria reported proper use of gloves $(14,23)$. Compared to this finding much lower proportion of respondents in studies in Hayatabad medical complex in Pakistan and Egypt use gloves with percentages $45 \%$ and $54.5 \%$ respectively $(15,24)$. This dissimilarity may be due to differences in working setup, the one done in Pakistan included other HCWs and that of the Bangladesh and Nigeria studies asked respondents habit of glove use while caring for any patient. The number of respondents involved in the Pakistan and Nigerian studies were also low that might contribute to this discrepancy $(14,23,24)$.

Significant proportion of nurses took special measures to protect self from these infectious diseases. In the present study $49.1 \%$ always and $25.1 \%$ usually use additional infection prevention mechanisms. Marking the status on the chart is one of the non-recommended practices done by many HCWs to give special precaution for that patient. In the present study about half ( $52 \%$ ) always and $20.9 \%$ usually practice it. In the study from Kutahya, majority (84.3\%) of the HCWs use additional infection prevention precautions and $69.3 \%$ of the preferred wearing double gloves for treating hepatitis $C$ patient having bleeding (17). In the study form Pakistan $7 \%$ of the respondents need to treat hepatitis B patients in isolated room and $65 \%$ replied as they use separate dressing set for these patients (24). In the Ghanaian study also $10.4 \%$ always do carefully with hepatitis B patients (25). Fear of acquiring infection and discriminating patients with hepatitis $B$ and $C$ viruses is most probably created from easy transmission of these viruses and lack of appropriate treatment.

Only $28.7 \%$ of nurses participated in this study always deliver the same standard of care for patients infected with hepatitis B and C. Moreover $38.9 \%$ and $38.4 \%$ of the participants always respond to these patients needs and talk positively about them respectively. The study from Pakistan also revealed that $68.9 \%$ of nurses need to deliver equal standard of care for hepatitis $C$ patient (26). This percentage may be elevated because it was their perception towards hypothetical patient not what they were performing. 
Significantly low proportion of the respondents (35.8\%) in this study had good practice towards patients with hepatitis B and C viruses. Higher percentage of respondents from studies in Dhaka medical college (87.4\%), Bangladesh (49.3\%), and Jimma (40.9\%) were found to have good preventive practice $(14,16,27)$. These figures were higher because they assess nurses and other HCWs practice of standard precautions to prevent hepatitis $B$ and $C$ infections while the current study assessed nurse's practices beyond this.

This study also examined factors associated with nurse's attitude and practice towards hepatitis B and C patients. Nurses who attended training on infection prevention were also 2.1 times more likely to be positive for hepatitis $B$ and $C$ patients than who didn't get training on infection prevention [AOR: $2.1 ; 95 \mathrm{Cl}$ (1.325-3.418)]. This may be because getting training on infection prevention increases nurses knowledge on how to treat a patient with hepatitis safely and confidently which in turn affects attitude and practice (14). Getting hepatitis B vaccination also found to increase the likely to have positive attitude by 6.6 [OR: $6.6 ; 95 \% \mathrm{Cl}(2.78-17.28)]$. The possible reason for this may be vaccination decreases the fear of acquiring infection and increases confidence in protecting self that many nurses lack.

Nurses worked for more than 10 years were 3.8 times more likely to have good practice than those who worked for $1-5$ years [AOR: $3.8 ; 95 \% \mathrm{Cl}(1.31-10.98)]$. A study done in Hayatabad medical complex in Pakistan found that those who worked for more than 4 years better use gloves (24). Nurses sustained needle stick injury from hepatitis $B$ and $C$ patients had 5.2 times poor practice than who respondents didn't sustained it [AOR: $5.2 ; 95 \% \mathrm{Cl}(1.236-21.862)$ ]. Vaccination against hepatitis B and positive attitude were found to have significant association with nurse's practice towards hepatitis $B$ and $C$ patients with P-values 0.006 and 0.000 respectively. The study from Nigeria also disclosed as positive attitude had significant association with good practice $(p=0.001)(37)$. This is because having positive attitude helps to take a safe and effective action.

\section{Limitations Of The Study}

One limitation of this study was the self-reported method of assessment of nurse's practices towards patients with hepatitis $B$ and $C$ viruses; the level practice may have been better assessed by observation although that couldn't be done because of shorter duration to get large number of hepatitis $B$ and $C$ patients being cared by nurses. Some of the information requested in the data collecting tool may be under-reported due to recall bias. Furthermore, Cross sectional study design is also ill in identifying which variable causes a change to the other in the exposure outcome relationship.

\section{Conclusion}

It could be inferred from this study that the overall level of nurse's attitude and practice towards patients with hepatitis B and C viruses is significantly low. Even though nurses better comply with some of the standard precautions, they use additional infection control measures which are not recommended and may be of discriminatory. 
Nurses trained on infection prevention and vaccinated against hepatitis B were more likely to have positive attitude towards patients with hepatitis $B$ and $C$ viruses. Nurses who were positive to accept hepatitis $B$ and $C$ patients deliver better acceptable care than those who had negative attitude. Respondents worked for more than 10 years and those vaccinated against hepatitis $B$ found to have good level of practice. Whereas, previous needle stick injury from hepatitis $B$ and $C$ patients influenced the respondent's practice to be poor.

\section{Abbreviations}

AOR: Adjusted Odds Ratio; BBF: Blood and Body Fluid; Cl: Confidence Interval; COR: Crude Odds Ratio; ETB: Ethiopian Birr; FMOH: Federal Ministry Of Health; HBV: Hepatitis B Virus; HCV: Hepatitis C Virus; HCW: Health Care Worker; PPE: Personal Protective Equipment; TASH: Tikur Anbesa Specialized Hospital

\section{Declarations}

\section{Ethics approval and consent to participate}

Letter of approval was obtained from institutional review board of Addis Ababa University, College of health sciences, school of Nursing and midwifery with protocol number 028/19/SNM. Additionally, letter of permission was obtained from Addis Ababa health bureau with issue number 36048/227. Written consent was obtained from study participants after informing them about the study.

Competing interest: The authors declare that they have no conflicts of interest regarding this manuscript publication in this journal.

Availability of data and material: The data used in this manuscript is available upon reasonable request by contacting the corresponding author via email address.

Funding: The corresponding author confirmed that financial funding was received from Debre Tabor University for the study but, not received any fund for publication of this article.

Authors' contributions: All authors contributed to the design of the study and the interpretation of data. TA and ZA performed the data analysis and compiled the work. TA and AT drafted the manuscript. All authors critically revised the draft manuscript as well they read and approved the final manuscript.

\section{Acknowledgments}

We would like to thank Debre Tabor university for funding this study. We are grateful to authorities of the selected hospitals for permitting us collect data from participants. We also appreciate the study participants for providing their valuable time and information.

\section{References}


1. Global hepatitis report 2017. Geneva: World Health Organization, 2017.

2. Belyhun, Yeshambel, Melanie Maier AM. Hepatitis viruses in Ethiopia_ a systematic review and metaanalysis. BMC Infect Dis. 2016;16(761).

3. Pfaender, Stephanie, Fabian A. Helfritz AS. Environmental Stability and Infectivity of Hepatitis C Virus (HCV) in Different Human Body Fluids. Front Microbiol. 2018;9(504).

4. Lindqvist B, Holmberg A, Bla J. High levels of hepatitis B virus DNA in body fluids from chronic carriers. J Hosp Infect. 2006;64:352-7.

5. Samargandy, Shireen A. Lujain M. Bukhari SAS. Epidemiology and clinical consequences of occupational exposure to blood and other body fluids in a university hospital in Saudi Arabia. Saudi Med J. 2016;37(7):783-790.

6. Yasin, Jemal, Roman Fisseha FM. Occupational exposure to blood and body fluids and associated factors among health care workers at the University of Gondar Hospital, Northwest Ethiopia. Env Heal Prev Med. 2019;24(18).

7. Guidelines for the prevention, care and treatment of persons with chronic hepatitis B infection. Geneva: World Heal Organization, 2015.

8. WHO. Guidance on Prevention of viral Hepatitis B and c among people who inject druss. 2012;

9. Combating hepatitis B and C to reach elimination by 2030. Geneva: World Heal Organization, 2016.

10. Prevention, care and treatment of viral hepatitis in the African region: framework for action, 2016 2020. Geneva: World Heal Organization, 2017.

11. Shiferaw, Fassil, Mekitew Letebo AB. Chronic viral hepatitis_ policy, regulation, and strategies for its control and elimination in Ethiopia. BMC Public Health. 2016;16(769).

12. Ebrahim ZT. A qualitative study on individual experiences of chronic hepatitis B patients - Taheri Ezbarami - 2017 - Nursing Open - Wiley Online Library. 2017.

13. Wada K, Smith DR, Ishimaru T. Reluctance to care for patients with HIV or hepatitis B / C in Japan. BMC Pregnancy Childbirth. 2016;1-6.

14. Mehriban N, Ahsan GU, Islam T. Knowledge and preventive practices regarding Hepatitis B among nurses in some selected hospitals of Dhaka city , Bangladesh. South East Asia J Public Heal. 2014;4(1):48-52.

15. Shoman AE, Salama SI, Sayed AM, Eldin WS, Fathy AM, Fouad WA, et al. Knowledge Health Care and Practice of Physicians, Nurses and Traditional Providers about Hepatitis C Virus Infection in Six Egyptian Governorates. Egypt J Community Med. 2015;33(4):51-65.

16. Gemeda DH, Abdusemed KA. Hepatitis B and C Viral Infection: Prevalence, Knowledge, Attitude, Practice, and Occupational Exposure among Healthcare Workers of Jimma University Medical Center, Southwest Ethiopia. Sci World J. 2019;

17. Korkmaz, Pınar, Cemile Uyar, Ahmet Ozmen OT. Knowledge and attitude of health care workers toward patients with hepatitis C infection. Southeast Asian J Trop Med Public Heal. 2016;47(5). 
18. Ishimaru T, Wada K, Thi H, Hoang X, Thi A, Bui M, et al. Nurses ' willingness to care for patients infected with HIV or Hepatitis B / C in Vietnam. Environ Health Prev Med. 2017;22(9):1-7.

19. Ishimaru, Tomohiro, Koji Wada, Sara Arphorn DRS. Barriers to the acceptance of work colleagues infected with Hepatitis B and Hepatitis C in Japan. J Occup Heal. 2016;58(3).

20. Villar, Livia M., Glaucia S. Silva VS de P. Assessment of Hepatitis B and Hepatitis C Knowledge and Attitudes in a Sample of Health Care Workers from Southeast Brazil. Virus Rev Res. 2017;22.

21. Abdela A, Woldu B, Haile K, Mathewos B, Deressa T. Assessment of knowledge, attitudes and practices toward prevention of hepatitis $B$ virus infection among students of medicine and health sciences in Northwest Ethiopia. BMC Res Notes. 2016;9(1).

22. Ishimaru T, Wada K, Thi H, Huong X, Thi B, Anh M, et al. Nurses ' attitudes towards co-workers infected with hiv or hepatitis b or c in Vietnam. Southeast Asian J Trop Med Public Heal. 2017;48(2).

23. Bello FM, Health P, Anne CP. Health Workers ' Knowledge, Attitude and Practice towards Hepatitis B Infection in Northern Nigeria. Int J Caring Sci. 2016;9(3):939-54.

24. Kamran Hakeem Khan, Ayaz Ayub, Hamid Hussain, Iftikhar Qayyum ANN. Knowledge attitude and practice regarding Hepatitis $B$ infection among hospital staff-nurses in Hayatabad Medical Complex Peshawar, KPK. KMUJ. 2017;8(2).

25. Afihene MY, Duduyemi BM, Khatib M. Knowledge, attitude and practices concerning Hepatitis B infection, among healthcare workers in Bantama, Ghana: a cross sectional study. Int J Community Med Public Heal. 2015;2(3):244-53.

26. Muhammad Hussain, Muhammad Afzal, Farzana Bibi, Muqadus Tasneem, Muhammad Rafique I. Knowledge and attitude level of nurses about Hepatitis C patient. Saudi J Med Pharm Sci. 2017;3(7):681-92.

27. $\mathrm{Nr} \mathrm{K}, \mathrm{Ha}$ M. Knowledge and preventive practice regarding hepatitis B among the nurses in Dhaka medical college hospital. J Dhaka Med Coll. 2017;26(1):5-11.

28. Kamran Hakeem Khan, Ayaz Ayub, Hamid Hussain, Iftikhar Qayyum ANN. Knowledge attitude and practice regarding Hepatitis $B$ infection among hospital staff-nurses in Hayatabad Medical Complex Peshawar, KPK. KMUJ. 2016;8(2):90-4. 